\title{
Caracterización de nueve agroecosistemas de café de la cuenca del río Porce, Colombia, con un enfoque agroecológico
}

\author{
The categorization of nine coffee agroecosystems from the Porce river basin, \\ Colombia, from an agroecological perspective
}

\author{
Mónica María Machado Vargas ${ }^{1}$, Clara Inés Nicholls ${ }^{1}$, Sara María Márquez, \\ Sandra Turbay ${ }^{*}$
}

\section{RESUMEN}

El propósito de este artículo es caracterizar nueve agroecosistemas de café de los Andes colombianos con un enfoque agroecológico. Los atributos y los indicadores evaluados son agrupados en tres dimensiones: económica, social y técnico-productiva. Los indicadores son sensibles a las condiciones del agroecosistema y pueden ser fácilmente comprendidos por los agricultores. La baja productividad en los cafetales y el riesgo económico son identificados como los principales problemas. La seguridad alimentaria tiene valores altos en la mayor parte de las fincas a pesar de las deficiencias en la productividad del café. La metodología propuesta podría ser adaptada por otros investigadores para caracterizar agroecosistemas campesinos sin olvidar que la selección de los indicadores debe ser el resultado de un proceso participativo; los indicadores usados en este trabajo no dan cuenta de todas las condiciones internas y externas que afectan los agroecosistemas, pero destacan factores relevantes para la reproducción social de las familias campesinas.

Palabras clave: agroecología, indicadores, café, agricultura familiar, agroecosistemas.

\begin{abstract}
The purpose of this paper is to characterize nine coffee agroecosystems from the Colombian Andes with an agroecological approach. Attributes and indicators evaluated are grouped under three dimensions: economic, social and technical-productive. Indicators are sensitive to agroecosystem conditions and can be easily understood by farmers. Low productivity of coffee plantations and economic risk are identified as the main problems. The study found high food security in most of the farms despite deficiencies in coffee productivity. The methodology proposed could be adapted by other researchers for the characterization of peasant agroecosystems without forgetting that setting indicators should be a participatory process; indicators used in this work do not account for all internal and external conditions that affect agroecosystems but they highlight relevant factors for social reproduction of peasant families.
\end{abstract}

Key words: agroecology, indicator, coffee, family farming, agroecosystems.

\section{Introducción}

Uno de los desafíos que enfrentan tanto agricultores como extensionistas e investigadores es saber en qué estado de salud se encuentra el agroecosistema. Por esto, algunos especialistas (Hart, 1985; Masera et al., 1999; Astier et al., 2008; Sarandón, 2002) han diseñado metodologías para la caracterización del agroecosistema que permiten monitorear su evolución y proponer rediseños agroecológicos. Para la caracterización se suelen determinar unos indicadores representativos que pueden ser cualitativos o cuantitativos. Un indicador es una señal que apunta a una determinada condición, es decir, su objetivo está enfocado a la existencia de riesgos, potencialidades y tendencias en el desarrollo de un determinado territorio o comunidad para orientar a la toma de decisiones (Pereira y Quacchia, 2009; Sarandón y Flores, 2009). El indicador representa una variable que asume un valor en un tiempo determinado; a su vez, una variable es una representación de un atributo en un determinado sistema. No existen indicadores universales, estos deben ser ajustados o definidos de acuerdo con

1 Grupo de investigación Medio Ambiente y Sociedad, Universidad de Antioquia. Sede de Investigación Universitaria, torre 1, oficina 213, tel. 57-4-2196480, Medellín, Colombia.

* Autor para correspondencia: sandra.turbay@udea.edu.co 
la realidad del estudio y el problema a evaluar. Cada sistema es único y los criterios o indicadores elegidos para caracterizar un sistema pueden no ser relevantes para otros (Sarandón y Flores, 2009). Los indicadores para una caracterización sistémica deben reflejar además la integración de aspectos sociales, ambientales y económicos (Pereira y Quacchia, 2009; Sarandón y Flores, 2009).

La preocupación por la sostenibilidad de la agricultura ha hecho que no se tengan en cuenta indicadores únicamente técnicos o económicos del estado de los agroecosistemas. La agroecología, por ejemplo, considera dimensiones ambientales, económicas, políticas y culturales de los sistemas productivos y proporciona principios ecológicos básicos para estudiar, diseñar y manejar agroecosistemas que ofrecen una alternativa a los impactos ecológicos, económicos y sociales de la agricultura convencional (Altieri, 1999; Rosset et al., 2000). La agroecología se propone aumentar la productividad sobre el terreno, reducir la pobreza rural, mejorar la nutrición de las familias campesinas y aumentar la resiliencia frente al cambio climático (De Schutter, 2010).

Este artículo se propone ilustrar la aplicación de una metodología para la caracterización de los agroecosistemas con enfoque sistémico que fue empleada en nueve fincas cafeteras de la cuenca del río Porce, Colombia. Aunque el artículo presenta en forma resumida los resultados de la caracterización, el interés principal del mismo es ofrecer alternativas a investigadores y extensionistas rurales para hacer diagnósticos rápidos en forma holística y hacer seguimiento a los cambios que tiene un agroecosistema, vinculando en el proceso a los agricultores.

\section{Materiales y Métodos}

\section{Descripción del área de estudio}

El estudio se llevó a cabo en la cuenca del río Porce sobre la cordillera central de los Andes. La topografía de la cuenca es irregular y pendiente y presenta altitudes que oscilan entre 80 y los $3.320 \mathrm{msnm}$ (CTA \& COSUDE, 2013). Este estudio se concentró en los municipios de Amalfi, Gómez Plata, Santa Rosa, Santo Domingo y Yolombó. Las fincas estudiadas están en el área de influencia de la hacienda Vegas de la Clara de la Universidad de Antioquia, y entre los 1.025 y los $1.500 \mathrm{msnm}$. De acuerdo con la clasificación ecológica de Holdridge,
(1978) la zona de vida es bosque muy húmedo premontano (bmh-PM) con transición al húmedo tropical. Los suelos de esta zona fueron clasificados por Jaramillo (1989) como Ustoxic Dystropept, suelos poco evolucionados que se derivan principalmente de cuarzo-diorita, una roca ígnea intrusiva perteneciente al Batolito antioqueño. La temperatura promedia anual es de $19{ }^{\circ} \mathrm{C}$ a $22^{\circ} \mathrm{C}$. El régimen de lluvias es bimodal, con dos períodos secos y dos lluviosos; el promedio anual de precipitaciones oscila entre los 2.500 y $3.000 \mathrm{~mm}$ anuales. La economía regional actual está orientada a la producción para la subsistencia. La ganadería extensiva se realiza en grandes predios en las vegas del río Porce, mientras que la agricultura campesina se desarrolla en las pendientes de la cordillera. La producción panela a pequeña escala en trapiches artesanales es una actividad económica muy importante. En la zona de estudio la principal organización de productores es la Federación Nacional de Cafeteros de Colombia.

\section{Metodología para la caracterización del agroecosistema de café}

Para la caracterización del agroecosistema de café se estructuraron las siguientes etapas adaptadas de la metodología de Sarandón (2002) y Sarandón et al. (2006): selección de los agricultores participantes; definición de las dimensiones y los atributos de análisis; construcción de los indicadores a evaluar; recolección de datos; integración de los resultados y, finalmente, determinación de los puntos críticos.

\section{Selección de los agricultores participantes}

En los cinco municipios se realizaron recorridos por las fincas, encuestas y entrevistas semiestructuradas a 48 agricultores familiares, con ellos se hicieron reuniones grupales para la selección de nueve agricultores, que serían objeto de la caracterización de sus fincas y de una propuesta de rediseño agroecológico. En la Tabla 1 se muestran los criterios de selección de las fincas. Los campesinos evaluaron las fincas asignando a cada criterio un valor entre cero y uno. Al final se seleccionaron los predios que obtuvieron los mejores puntajes, indicando con esto que eran los que cumplían con el mayor número de criterios. Los tres últimos criterios $(13,14$ y 15$)$ se plantearon como criterios adicionales en caso que se presentara un empate. Se escogieron nueve 
fincas teniendo en cuenta el carácter limitado de los recursos y del tiempo y porque la intención era que estas fincas debían convertirse en faros para que su experiencia fuera replicada en las demás unidades agrícolas familiares.

\section{Definición de las dimensiones y los atributos de análisis para la caracterización}

Se seleccionaron tres dimensiones y para cada una de ellas se escogieron unos atributos que se describen a continuación:

\section{Dimensión económica}

La agroecología propone una agricultura ligada con el medio ambiente que también sea económicamente viable, es decir, que pueda proveer de seguridad alimentaria, que tenga alto rendimiento y productividad y que disminuya los riesgos económicos. Los atributos que se evaluaron fueron:

- Seguridad alimentaria: la seguridad alimentaria no implica necesariamente la producción de alimentos en la finca pero sí su disponibilidad. Esta depende del acceso a los centros de distribución de alimentos y de los ingresos que percibe el grupo familiar. Sin embargo, la producción de alimentos a pequeña escala puede ser una alternativa para una adecuada alimentación en el hogar además de aumentar los ingresos por la vía de la comercialización y el autoabastecimiento (Álvarez et al., 2007).

Tabla 1. Matriz de criterios de selección de las nueve fincas de los municipios de Gómez Plata, Amalfi, Santa Rosa, Santo Domingo y Yolombó, 2011.

\begin{tabular}{|c|c|c|}
\hline $\mathrm{N}$ & Criterio & Soporte del criterio \\
\hline 1. & Tamaño del predio & $\begin{array}{l}\text { No debe ser inferior a una hectárea. La finca, bien rediseñada, debe permitir entre uno y } \\
\text { dos salarios mínimos legales vigentes. }\end{array}$ \\
\hline 2. & $\begin{array}{l}\text { Composición del grupo } \\
\text { familiar }\end{array}$ & $\begin{array}{l}\text { Que el agricultor(a) viva con su familia en la finca por la importancia de la fuerza de } \\
\text { trabajo familiar. }\end{array}$ \\
\hline 3. & $\begin{array}{l}\text { Implementación de } \\
\text { prácticas ecológicas }\end{array}$ & $\begin{array}{l}\text { Que tenga al menos cuatro prácticas ecológicas como compostera, asociación de cultivos, } \\
\text { rotación, conservación de aguas, conservación de suelos y huerta familiar. }\end{array}$ \\
\hline 4. & $\begin{array}{l}\text { Tiempo de dedicación a } \\
\text { la finca }\end{array}$ & Que el agricultor(a) trabaje en su finca tiempo completo. \\
\hline 5. & Tenencia de la tierra & $\begin{array}{l}\text { Que el agricultor sea propietario de su tierra o que por lo menos esté en proceso de sucesión; } \\
\text { que la finca no sea prestada ni alquilada. }\end{array}$ \\
\hline 6. & $\begin{array}{l}\text { Actividades diversas } \\
\text { generadoras de ingresos }\end{array}$ & $\begin{array}{l}\text { La finca debe tener al menos cuatro actividades generadoras de ingresos continuos (no } \\
\text { esporádicos). }\end{array}$ \\
\hline 7. & Ubicación de la finca & Que no quede a más de 45 minutos de la cabecera municipal. \\
\hline 8. & Participación comunitaria & $\begin{array}{l}\text { Que el agricultor(a) haga parte de algún tipo de organización de base (junta de acción } \\
\text { comunal, junta de acueducto, concejo municipal de desarrollo, grupos juveniles, de mujeres, } \\
\text { etc.) y que con preferencia tenga parte en las directivas. }\end{array}$ \\
\hline 9. & Experiencia productiva & $\begin{array}{l}\text { Que haya sido agricultor durante los últimos cinco años y que tenga alguna experiencia en } \\
\text { algún tipo de agricultura alternativa. }\end{array}$ \\
\hline 10. & Disponibilidad de agua & Que exista agua en la finca y que haya disponibilidad para los animales y para riego. \\
\hline 11. & Créditos & Que no tenga más de dos créditos agropecuarios vigentes. \\
\hline 12. & Utilización de agrotóxicos & De uso moderado, con tendencia a nulo. \\
\hline 13. & Tecnologías apropiadas & $\begin{array}{l}\text { Que tenga implementadas en su finca cuatro tecnologías apropiadas como ariete, marquesina, } \\
\text { biodigestor, estufa eficiente, filtros de agua, agrotanques, beneficiadero ecológico, etc. }\end{array}$ \\
\hline 14. & $\begin{array}{l}\text { Articulación producción } \\
\text { comercialización }\end{array}$ & $\begin{array}{l}\text { Se busca que el agricultor venda sus productos al consumidor final o al menos a puntos de } \\
\text { distribución como cooperativas, asociaciones, supermercados y tiendas. }\end{array}$ \\
\hline 15. & Resolución de conflictos & Que el agricultor no fuera causante de conflictos en la comunidad. \\
\hline
\end{tabular}


Para evaluar la seguridad alimentaria de las familias se realizó una encuesta a la persona encargada de preparar los alimentos en la familia.

- Rendimiento: para que una agricultura sea económicamente viable es necesario que haya buenos rendimientos, para evaluarlos se utilizó el indicador $\mathrm{kg} / \mathrm{ha} / \mathrm{año}$, tomando como referente el rendimiento de la variedad Castillo según Federación Nacional de Cafeteros de Colombia.

- Riesgo económico: una mayor diversidad de cultivos permite un mejor uso de los recursos en el agroecosistema. Las tecnologías de bajos insumos reducen la dependencia de insumos externos (Gliessman, 1998; Altieri, 2002). Al comercializar más de un producto el productor disminuye el riesgo económico.

\section{Dimensión técnica productiva}

El estado del cultivo de café y la calidad del suelo se evaluaron con una metodología propuesta por Altieri y Nicholls (2002). Para evaluar la fertilidad del suelo se realizó un análisis de suelos en los laboratorios de la Universidad Nacional de Colombia.

- Calidad del suelo: definida como la capacidad del suelo de funcionar dentro de los límites de un ecosistema, sustentando la productividad biológica y promoviendo la salud de las plantas y animales (Conceição et al., 2005).

- Salud del cultivo: los indicadores seleccionados que permiten identificar el estado fitosanitario de un cultivo son apariencia y crecimiento, resistencia o tolerancia al estrés, incidencia de plagas y enfermedades y competencia por arvenses. La diversidad genética y de especies cultivadas (espacio y tiempo), así como la vegetación circundante, son fundamentales en la salud del cultivo, ya que las interacciones entre los varios componentes bióticos y abióticos, mediante el ensamble de una biodiversidad funcional, incrementa las oportunidades para la coexistencia e interacción benéfica entre las especies y mejora la sustentabilidad del agroecosistema (Altieri, 2002).

\section{Dimensión social}

La agroecología propone una agricultura que dignifique la vida en el campo con un desarrollo rural integral. Para evaluar la dimensión social se escogieron dos indicadores:

- Tenencia de la tierra: la propiedad de la tierra es una garantía de estabilidad, reduce los costos de producción y constituye un elemento esencial en la reproducción social de la familia campesina.

- Redes sociales: el tejido social es fuente de mano de obra, información, servicios y apoyo en momentos de crisis.

\section{Construcción de los indicadores a evaluar}

Con los nueve agricultores se realizaron talleres de formación y discusión acerca de los principios de la agroecología, las limitaciones técnicas en sus cafetales y la selección de indicadores que a su modo de ver fueran relevantes para tener una visión global del estado de los nueve agroecosistemas (Tabla 2). Los indicadores debían cumplir con los siguientes requisitos: ser fáciles de medir, comprensibles para alguien no experto, ser capaces de reflejar los procesos que ocurrían dentro de los agroecosistemas y capaces de detectar tendencias en el ámbito de finca (Conceição et al., 2005; Carneiro et al., 2007; Sarandón, 2002). Los indicadores que se utilizaron fueron de estado, es decir, que aportaban información referente a la situación del sistema en el momento del estudio (Sarandón, 2002). La escala que se utilizó para calificar cada indicador fue de 1 a 4 , siendo 4 el valor alto y 1 el valor muy bajo.

\section{Recolección de datos}

La recopilación de los datos se realizó por medio de entrevistas semiestructuradas y técnicas rápidas de análisis de fertilidad de suelos.

\section{Integración de los resultados}

Los resultados de la evaluación de los indicadores se resumieron, analizaron e integraron mediante la elaboración de un diagrama tipo radar, como lo recomiendan Astier et al. (2008), ya que permite mostrar las características cualitativas de la 
Tabla 2. Dimensiones, atributos e indicadores estratégicos utilizados para la caracterización de los nueve agroecosistemas de café en una escala de 1 a 4, cuenca del río Porce.

\section{DIMENSIÓN ECONÓMICA}

\begin{tabular}{|c|c|c|}
\hline Atributo & Indicador & Escala (1-4) \\
\hline \multirow[t]{4}{*}{ Seguridad alimentaria } & Diversidad de productos & $\begin{array}{l}\text { 1. Muy baja: un solo producto } \\
\text { 2. Baja: con dos productos } \\
\text { 3. Media: con tres productos } \\
\text { 4. Alta: con cuatro productos o más }\end{array}$ \\
\hline & Disponibilidad de alimentos & $\begin{array}{l}\text { 1. Muy baja: } 1-30 \% \\
\text { 2. Baja: del } 30 \%-40 \% \\
\text { 3. Media: } 40 \%-60 \% \\
\text { 4. Alta: del } 60 \%-100 \%\end{array}$ \\
\hline & Ingreso mensual & $\begin{array}{l}\text { 1. Muy bajo: de } 0-100.000 \text { pesos } \\
\text { 2. Bajo: de } 100.000-250.000 \text { pesos } \\
\text { 3. Medio: } 250000 \text { pesos a } 589.500 \text { pesos (un salario mínimo) } \\
\text { 4. Alto: } 589.000 \text { a } 1.179 .000 \text { pesos (dos salarios mínimos) }\end{array}$ \\
\hline & Autoconsumo & $\begin{array}{l}\text { 1. Muy bajo: } 1-30 \% \\
\text { 2. Bajo: del } 30 \%-40 \% \\
\text { 3. Medio: } 40 \%-60 \% \\
\text { 4. Alto: del } 60 \%-100 \%\end{array}$ \\
\hline Rendimiento & Producción/área/año & $\begin{array}{l}\text { 1. Muy baja: de } 1.000-2.000 \text { kilos } \\
\text { 2. Baja: de } 3.000-5.000 \text { kilos } \\
\text { 3. Media: de } 6.000-9.000 \text { kilos } \\
\text { 4. Alta: de } 10.000-12.000 \text { kilos }\end{array}$ \\
\hline \multirow[t]{3}{*}{ Riesgo económico } & Diversidad de ventas & $\begin{array}{l}\text { 1. Muy baja: un solo producto } \\
\text { 2. Baja: dos productos } \\
\text { 3. Media: tres productos } \\
\text { 4. Alta: cuatro a cinco productos }\end{array}$ \\
\hline & Vías de comercialización & $\begin{array}{l}\text { 1. Muy baja: un canal de comercialización } \\
\text { 2. Baja: dos canales } \\
\text { 3. Media: tres canales } \\
\text { 4. Alta: cuatro canales o más }\end{array}$ \\
\hline & Dependencia de insumos externos & $\begin{array}{l}\text { 1. Muy baja: } 100 \% \text { los insumos no son orgánicos y se compran } \\
\text { 2. Baja: } 80-60 \% \text { los insumos no son orgánicos } \\
\text { 3. Media: } 60-20 \% \text { los insumos no son orgánicos } \\
\text { 4. Alta: } 100 \% \text { los insumos son orgánicos }\end{array}$ \\
\hline
\end{tabular}

DIMENSIÓN TÉCNICO-PRODUCTIVA

\begin{tabular}{ccc}
\hline Atributo & \multicolumn{1}{c}{ Indicador } & Escala (1-4) \\
\hline Calidad de suelo & Estructura del suelo & 1. Muy baja: sin estructura
\end{tabular}

2. Baja: sin estructura o granular, bloques, laminar, columnar y prismática, con grado débil, tamaño fino y poco consistente 3. Media: granos simples, granular, bloques, laminar, columnar y prismática, con grado moderado, tamaño medio y moderadamente consistente.

4. Alta: granular, bloques, laminar, columnar y prismática, con grado fuerte, tamaño grande y muy consistente.

Profundidad efectiva

1. Muy baja: suelos muy resistentes a la penetración, con alta pedregosidad o condiciones muy húmedas.

2. Baja: suelos resistentes a la penetración, moderadamente pedregosos o condiciones húmedas.

3. Media: suelos moderadamente resistentes, poco pedregosos, o poco húmedos.

4. Alta: suelos sin resistencia, sin pedregosidad, sin humedad. 


\begin{tabular}{rc}
\hline Atributo & Indicador \\
\hline Salud del cultivo & Estado de materia orgánica superficial
\end{tabular}

Cobertura del suelo

Control de erosión

Fertilidad del suelo

Apariencia y crecimiento del cultivo

Resistencia o tolerancia a estrés

Incidencia de plagas y enfermedades
Escala (1-4)

1. Muy baja: presencia generalizada de residuos poco descompuestos, de tamaño grande u olor desagradable.

2. Baja: presencia generalizada de residuos parcialmente descompuestos, de tamaño medio, sin olor desagradable. 3. Media: presencia generalizada de residuos moderadamente descompuestos, de tamaño medio a fino, sin olor desagradable. 4. Alta: presencia generalizada de residuos bien descompuestos, de tamaño fino, sin olor desagradable.

1. Muy baja: la totalidad del suelo está desnudo durante todo el año.

2. Baja: el suelo tiene una cobertura entre 1 y $40 \%$ de su área, durante seis meses del año.

3. Media: el suelo tiene una cobertura entre 40 y $80 \%$ de su área durante diez meses del año.

4. Alta: el suelo tiene una cobertura mayor del $80 \%$ de su área durante más de diez meses del año.

1. Muy bajo: más de $40 \%$ de la zona de muestreo presenta cárcavas o canales o entre 75 y 100\% del horizonte A se ha perdido.

2. Bajo: entre el 20 y el $40 \%$ de la zona de muestreo presenta cárcavas o canales. Entre 50 y $75 \%$ del horizonte A se ha perdido.

3. Medio: entre el 5 y el $20 \%$ de la zona de muestreo presenta cárcavas o canales. Entre 25 y $50 \%$ del horizonte A se ha perdido.

4. Alto: no hay muestras de erosión.

1 Muy baja fertilidad del suelo.

2 Baja fertilidad del suelo.

3 Media fertilidad del suelo.

4 Alta fertilidad del suelo.

1. Muy bajos: más del $50 \%$ del cultivo presenta clorosis, deficiencia o desbalance nutricional y crecimiento pobre y poco denso.

2. Bajos: entre 20 y $50 \%$ del cultivo presenta clorosis u otro síntoma severo de deficiencia o desbalance nutricional y cultivo moderadamente pero no muy uniforme.

3. Medios: entre 1 y $20 \%$ del cultivo presenta clorosis u otro síntoma severo de deficiencia o desbalance nutricional y cultivo denso, no muy uniforme.

4. Altos: el cultivo no presenta ningún signo de clorosis o problema nutricional y buen crecimiento uniforme.

1. Muy baja: el cultivo no se recupera luego de un factor de estrés.

2. Baja: el cultivo se recupera poco después de un factor de estrés.

3. Media: el cultivo no se recupera totalmente luego de un factor de estrés, afectando parcialmente su desarrollo. 4. Alta: el cultivo se recupera totalmente luego de un factor de estrés.

1. Muy baja: más del $50 \%$ del cultivo muestra síntomas de daño por plagas o enfermedades.

2. Baja: entre 20 y $50 \%$ del cultivo muestra síntomas de daño por plagas o enfermedades.

3. Media: entre 5 y $20 \%$ del cultivo muestra síntomas de daño por plagas o enfermedades.

4. Alta: menos de 5\% del cultivo muestra síntomas de daño por plagas o enfermedades. 


\begin{tabular}{|c|c|c|}
\hline Atributo & Indicador & Escala (1-4) \\
\hline & Competencia por arvenses & $\begin{array}{l}\text { 1. Muy baja: las arvenses compiten con los cultivos en el } \\
\text { 100\% de los momentos críticos. } \\
\text { 2. Baja: las arvenses compiten con los cultivos en } 80 \% \text { de } \\
\text { los momentos críticos. } \\
\text { 3. Media: las arvenses compiten con los cultivos entre el } \\
50 \text { y } 80 \% \text { de los momentos críticos. } \\
\text { 4. Alta: las arvenses no están presentes en los momentos } \\
\text { críticos o están todo el tiempo del cultivo, pero no compiten. }\end{array}$ \\
\hline & Diversidad genética & $\begin{array}{l}\text { 1. Muy baja: solo una variedad por especie cultivada. } \\
\text { 2. Baja: dos variedades por especies cultivadas. } \\
\text { 3. Media: tres variedades por especie. } \\
\text { 4. Alta: más de tres variedades por especies cultivadas, } \\
\text { ninguna variedad domina más del } 50 \% \text { en el cultivo. }\end{array}$ \\
\hline & Diversidad de especies cultivadas & $\begin{array}{l}\text { 1. Muy baja: una sola especie cultivada. } \\
\text { 2. Baja: dos especies no asociadas donde una especie ocupa } \\
\text { el } 70 \% \text { del área cultivada, o dos especies asociadas donde } \\
\text { una predomina con más del } 70 \% \text { y ocupa estrato de cultivo. } \\
\text { 3. Media: dos especies no asociadas con máximo de } 70 \% \\
\text { del área por especie o dos especies asociadas donde ninguna } \\
\text { supera el } 70 \% \text { del área y por lo menos una es arbustiva. } \\
\text { 4. Alta: mayor dos especies no asociadas, con máximo de } \\
\text { 50\% del área por especie o mayor dos especies asociadas } \\
\text { donde ninguna supera el } 70 \% \text { del área y son herbáceas, } \\
\text { arbustivas y arbóreas. }\end{array}$ \\
\hline & Vegetación natural circundante & $\begin{array}{l}\text { 1. Muy baja: no hay áreas naturales en el agroecosistema } \\
\text { 2. Baja: menos del } 2 \% \text { del área total de la finca está cubierta } \\
\text { por vegetación natural, en forma de corredores y concentrada } \\
\text { en una sola parte de la finca. } \\
\text { 3. Media: entre } 2 \text { y } 4 \% \text { del área total está cubierta por } \\
\text { vegetación natural, en forma de corredores, franjas y parches } \\
\text { dispersos en varias partes de la finca. } \\
\text { 4. Alta: más del } 4 \% \text { del área total de la finca está cubierta } \\
\text { por vegetación natural, en forma de corredores, franjas y } \\
\text { parches dispersos en varias partes de la finca. }\end{array}$ \\
\hline
\end{tabular}

\begin{tabular}{|c|c|c|}
\hline \multicolumn{3}{|c|}{ DIMENSIÓN SOCIAL } \\
\hline Atributo & Indicador & Escala (1-4) \\
\hline \multirow[t]{5}{*}{ Redes sociales } & Tenencia de la tierra & $\begin{array}{l}\text { 1. Muy bajo: no tiene tierra } \\
\text { 2. Baja: tierra arrendada } \\
\text { 3. Medio: tiene carta de compra venta } \\
\text { 4. Alto: tiene título de propiedad }\end{array}$ \\
\hline & Apoyo de mano de obra de la familia & $\begin{array}{l}\text { 1. Muy bajo: contrata jornaleros } \\
\text { 2. Bajo: no hay apoyo } \\
\text { 3. Medio: tiene la ayuda de los hijos } \\
\text { 4. Alto: tiene el apoyo del cónyuge y los hijos }\end{array}$ \\
\hline & Presencia de parientes en la vereda & $\begin{array}{l}\text { 1. Muy baja: no tiene parientes en la vereda } \\
\text { 2. Baja: tiene solo hermanos } \\
\text { 3. Media: tiene solo padres } \\
\text { 4. Alta: tiene padres, hermanos y primos }\end{array}$ \\
\hline & Lazos de vecindad & $\begin{array}{l}\text { 1. Muy bajos: no tiene amigos } \\
\text { 2. Bajos: tiene visitas ocasionales } \\
\text { 3. Medios: lo visitan regularmente } \\
\text { 4. Altos: lo visitan mucho }\end{array}$ \\
\hline & Organización comunitaria & $\begin{array}{l}\text { 1. Muy bajo: no tiene apoyo institucional } \\
\text { 2. Bajo: poco apoyo institucional } \\
\text { 3. Medio: regular apoyo institucional } \\
\text { 4. Alto: buen apoyo institucional }\end{array}$ \\
\hline
\end{tabular}


evaluación de los indicadores y de cada uno de los agroecosistemas de café, mostrando la relación entre el valor real respecto del valor ideal.

\section{Determinación de los puntos críticos}

Con los resultados de los indicadores acerca del estado de los agroecosistemas de café se obtuvieron los puntos críticos que se calcularon mediante la diferencia entre los valores altos y los valores muy bajos.

\section{Resultados y Discusión}

En la Tabla 3 se describen, de manera sucinta, algunas características de las fincas estudiadas que son relevantes para comprender la dinámica de los agroecosistemas.

Tabla 3. Breve descripción de las nueve fincas seleccionadas del cañón del río Porce, nordeste antioqueño.

\begin{tabular}{|c|c|}
\hline \multicolumn{2}{|c|}{ Descripción de la unidad agrícola familiar } \\
\hline $\begin{array}{l}\text { Finca: Patio Bonito } \\
\text { Municipio: Santa Rosa de Osos } \\
\text { Propietario: Germán Hoyos }\end{array}$ & $\begin{array}{l}\text { Área: } 5 \mathrm{~h} \\
\text { Altura: } 1.300 \mathrm{msnm} \\
\text { Temperatura: } 22^{\circ} \mathrm{C} \\
\text { Cultivo principal: café (variedad Castillo y Colombia) } \\
\text { Asociación: plátano y yuca }\end{array}$ \\
\hline $\begin{array}{l}\text { Finca: El Cedro } \\
\text { Municipio: Santa Rosa de Osos } \\
\text { Propietario: Rafael Mesa }\end{array}$ & $\begin{array}{l}\text { Área: } 2 \mathrm{~h} \\
\text { Altura: } 1.500 \mathrm{msnm} \\
\text { Temperatura: } 20^{\circ} \mathrm{C} \\
\text { Cultivo principal: café (Castillo, Caturro y Colombia) } \\
\text { Asociación: plátano y yuca }\end{array}$ \\
\hline $\begin{array}{l}\text { Finca: Botero } \\
\text { Municipio: Amalfi } \\
\text { Propietario: Fabián Botero }\end{array}$ & $\begin{array}{l}\text { Área: } 3,5 \mathrm{~h} \\
\text { Altura: } 1.470 \mathrm{msnm} \\
\text { Temperatura: } 21-22^{\circ} \mathrm{C} \\
\text { Cultivo principal: café (Castillo y Caturro) } \\
\text { Asociación: guamo, plátano, aguacate y guanábanos }\end{array}$ \\
\hline $\begin{array}{l}\text { Finca: El Ocaso } \\
\text { Municipio: Amalfi } \\
\text { Propietario: Jhon Jairo Bonilla }\end{array}$ & $\begin{array}{l}\text { Área: } 0,36 \mathrm{~h} \\
\text { Altura: } 1.470 \mathrm{msnm} \\
\text { Temperatura: } 21-22^{\circ} \mathrm{C} \\
\text { Cultivo principal: café (Castillo, Catimoro y Caturro) } \\
\text { Asociación: guamo, plátano, aguacate y guanábanos. }\end{array}$ \\
\hline $\begin{array}{l}\text { Finca: Gramalote } \\
\text { Municipio: Yolombó } \\
\text { Propietario: Gloria Zabala }\end{array}$ & $\begin{array}{l}\text { Área: } 7 \mathrm{~h} \\
\text { Altura: } 1.400 \mathrm{msnm} \\
\text { Temperatura: } 22-25^{\circ} \mathrm{C} \\
\text { Cultivo principal: café (Catimore, Castillo y Colombia) } \\
\text { Asociación: plátano, guamo y nogal cafetero. }\end{array}$ \\
\hline $\begin{array}{l}\text { Finca: El Pomal } \\
\text { Municipio: Yolombó } \\
\text { Propietario: Mery Osorno }\end{array}$ & $\begin{array}{l}\text { Área: } 8 \mathrm{~h} \\
\text { Altura: } 1.320 \mathrm{msnm} \\
\text { Temperatura: } 22^{\circ} \mathrm{C} \\
\text { Cultivo principal: café (Pajarito, Castillo y Colombia) } \\
\text { Asociación: plátano, guamo, yarumo y nogal cafetero }\end{array}$ \\
\hline $\begin{array}{l}\text { Finca: El Pomal } \\
\text { Municipio: Gómez Plata } \\
\text { Propietario: Walter Sisquiarco }\end{array}$ & $\begin{array}{l}\text { Área: } 2,6 \mathrm{~h} \\
\text { Altura: } 1.500 \mathrm{msnm} \\
\text { Temperatura: } 20^{\circ} \mathrm{C} \\
\text { Cultivo principal: café (Rosario, Castillo y variedad 2000) } \\
\text { Asociación: plátano. }\end{array}$ \\
\hline $\begin{array}{l}\text { Finca: El Guanabano } \\
\text { Municipio: Gómez Plata } \\
\text { Propietario: Adrián Restrepo }\end{array}$ & $\begin{array}{l}\text { Área: } 40 \mathrm{~h} \\
\text { Altura: } 1.025 \mathrm{msnm} \\
\text { Temperatura: } 22-24{ }^{\circ} \mathrm{C} \\
\text { Cultivo principal: caña y café (Rosario, Castillo y } \\
\text { variedad 2000) } \\
\text { Asociación: plátano. }\end{array}$ \\
\hline $\begin{array}{l}\text { Finca: La Esperanza } \\
\text { Municipio: Santo Domingo } \\
\text { Propietario: Óscar Bustamante }\end{array}$ & $\begin{array}{l}\text { Área: } 7 \mathrm{~h} \\
\text { Altura: } 1.200 \mathrm{msnm} \\
\text { Temperatura: } 27^{\circ} \mathrm{C} \\
\text { Cultivo principal: café (variedad Castillo) } \\
\text { Asociación: sin asociación }\end{array}$ \\
\hline
\end{tabular}




\section{Dimensión económica}

En la Figura 1 se observa que para los atributos de seguridad alimentaria y riesgo económico el agricultor 5 (Óscar Bustamante) obtiene los mejores puntajes, esto se debe a que tiene una alta variedad de cultivos (café, cacao, caña de azúcar y frutales) que comercializa por diferentes vías. Además, gracias a las especies menores como aves y los peces tiene una mejor seguridad alimentaria. Aunque el rendimiento del café es bajo, esto no representa un riesgo económico porque los otros cultivos le permiten equilibrar la productividad. El agricultor con mayor riesgo económico es el 3 (Walter Sisquiarco), debido a que solo comercializa café y este tiene bajo rendimiento. Sin embargo, tiene una seguridad alimentaria media porque produce algunos alimentos para el autoconsumo.

Los demás agricultores están bien en seguridad alimentaria, pero tienen rendimientos bajos en el cultivo de café y están en situación de riesgo económico. Las familias producen el $30 \%$ de los lácteos, el $80 \%$ de los huevos y el $90 \%$ de las legumbres, los tubérculos, las verduras y hortalizas y las frutas que consumen. Las familias tienen tres o más ingestas al día, aunque el $60 \%$ de ellas tiene ingresos que no superan un salario mínimo legal vigente y el $40 \%$ restante no alcanza los dos salarios mínimos.

Los resultados evidencian que la seguridad alimentaria no depende exclusivamente de los ingresos monetarios de las familias. Aunque el riesgo económico sea alto, las familias tienen asegurada una buena alimentación. Presentan un riesgo económico mayor las familias que solamente tienen café para ofrecer en el mercado, las que no tienen rentas, las que no diversifican su economía y las que dependen de un único canal de comercialización. La venta de café está asegurada por la Federación Nacional de Cafeteros de Colombia, pero su precio puede variar sustancialmente de un año a otro. El endeudamiento por la compra de fertilizantes químicos es otro factor importante de riesgo. En Colombia los costos de fertilización han aumentado del $10 \%$ al $20 \%$ en los últimos años (Khalajabadi, 2008), y en el caso de la producción de café convencional el uso de fertilizantes puede representar hasta el 55\% de los costos totales (Castro-Tanzi et al., 2012). La principal fuente de materia orgánica en estas fincas es la pulpa de café, pero ningún agricultor la usa. Según Khalajabadi (2008), el uso de la pulpa descompuesta en dosis de $6 \mathrm{~kg} / \mathrm{planta} /$ año en el período de crecimiento vegetativo del café ha demostrado tener efectos benéficos en la primera cosecha.

Evaluación económica en nueve fincas del nordeste antioqueño

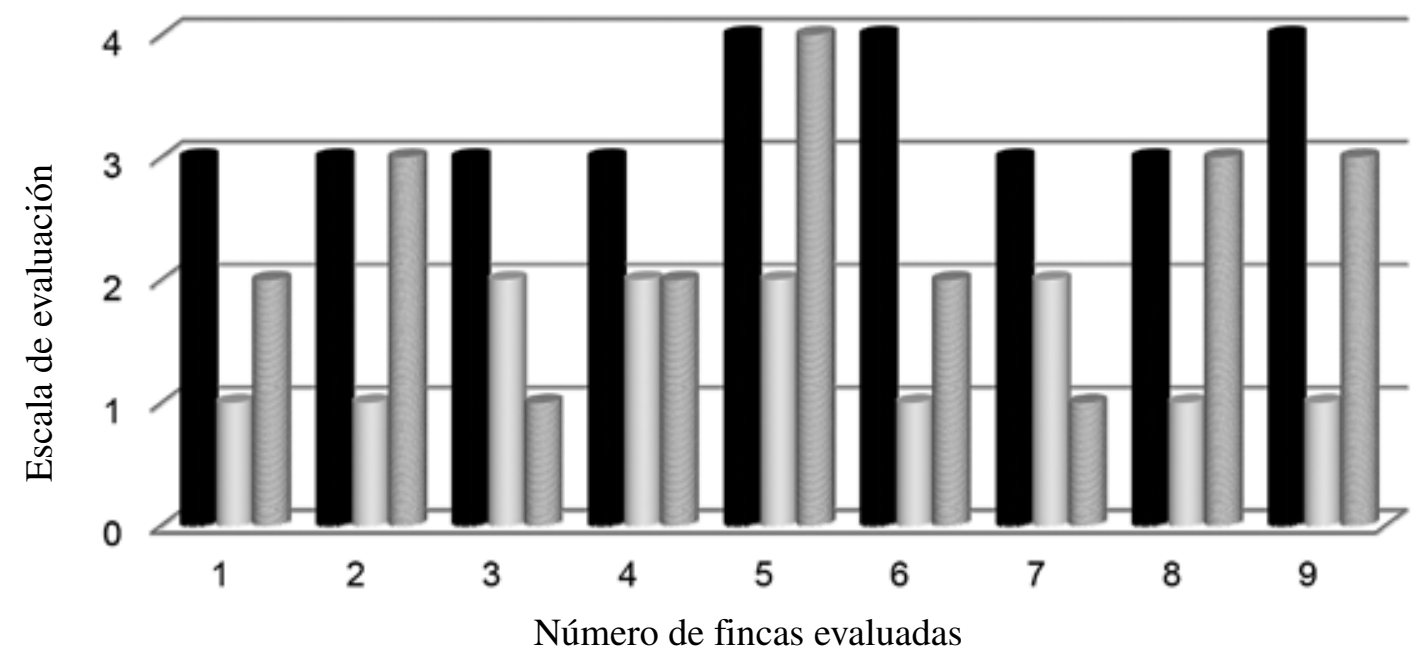

- A. Seguridad alimentaria

B. Rendimiento

요 D. Riesgo económico

Figura 1. Caracterización de la dimensión económica de los nueve agroecosistemas de café (1: Fabián Bonilla, 2: Jhon Jairo Bonilla.; 3: Walter Sisquiarco; 4: Adrián Ríos.; 5: Óscar Bustamante; 6: Germán Hoyos; 7: Rafael Mesa; 8: Mery Osorno.; 9 : Gloria Zabala) de la cuenca del río Porce. 
En todas las fincas estudiadas los rendimientos en la producción de café son bajos, lo que se explica en parte porque a finales del 2012 y principios del 2013, cuando se realizaron las encuestas, se presentó una sequía prolongada como efecto del evento de El Niño (IDEAM 2012, 2013), que en la zona cafetera está asociado a la deficiencia hídrica en el suelo. La floración se ve afectada y durante la etapa de llenado de los granos, que es crítica para la formación del fruto, se puede formar granos flotantes (vacíos) que producen pasilla (grano de menor calidad) (Cenicafé, 2009). La baja productividad es un llamado de atención para que los agricultores mejoren el manejo de sus cafetales.

\subsection{Dimensión técnico-productiva}

Como se puede observar en la Figura 2, los agricultores obtuvieron valores medios para la calidad del suelo y salud del cultivo con excepción de los agricultores 5 y 8 que obtienen valores más altos. El agricultor 5 (Óscar Bustamante) usa abonos verdes y abonos orgánicos. Para la mayoría la salud del cultivo tiene una calificación media, debido a la baja diversidad espacial del cultivo de café y a que solo se tiene una variedad de este. La agricultora 8 (Mery Osorno) registró los valores más altos en calidad del suelo y salud del cultivo, debido a la asociación de cultivos, al uso de abonos verdes y a la alta diversidad genética; además, la finca se encuentra en una matriz de vegetación natural que sirve como refugio de enemigos naturales de las plagas de los cultivos.

Los nueve agricultores evaluados tienen buenas prácticas agrícolas como son el deshierbe para controlar arvenses, el empleo de abonos verdes o mulch (restos vegetales de las podas se dejan en el suelo), que les permiten aumentar la materia orgánica y el mantenimiento de cobertura en el suelo, esto evita la erosión, aumenta la actividad biológica del suelo y mejorará su estructura. Siete de los nueve agricultores cultivan café bajo semisombrío, lo que trae algunos beneficios para el agroecosistema. Los árboles de sombrío también ejercen marcada influencia relativo a las condiciones del suelo, aumentando la materia orgánica y el reciclaje de nutrientes, economizan agua, disminuyen el desarrollo de las arvenses, ayudan a mantener el suelo agregado, impiden su movimiento, aumentan la porosidad del suelo favoreciendo la infiltración y absorción del agua y disminuyen la escorrentía minimizando las pérdidas del suelo, entre otros (Cenicafé, 2010). Los árboles de sombrío aportan altas cantidades de materia orgánica rica en lignina que al descomponerse mejora las condiciones físicas, químicas y biológicas del suelo (Khalajabadi, 2008). El uso de árboles fijadores de nitrógeno aumenta la descomposición de la hojarasca y la materia orgánica del suelo (Youkhana e Idol, 2009). El café bajo sombra, con una densidad de árboles adecuada,

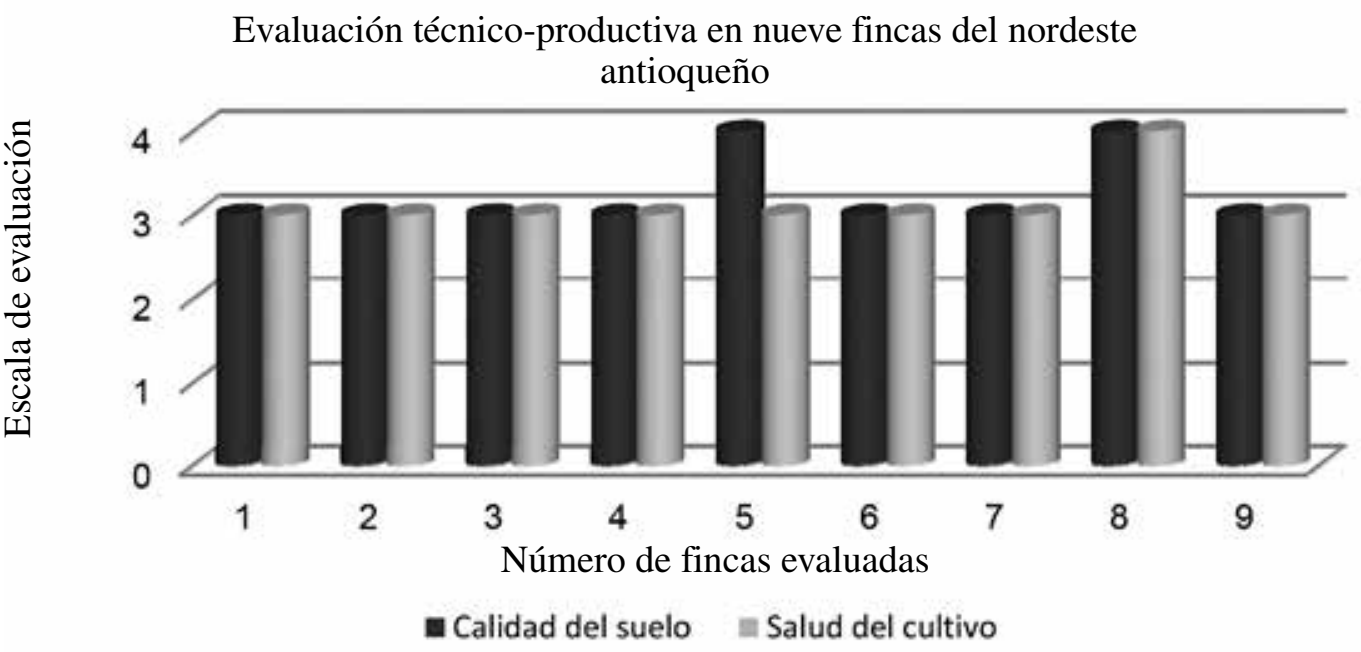

Figura 2. Caracterización de la dimensión ecológica y técnico-productiva de los nueve agroecosistemas de café (1: Fabián Bonilla, 2: Jhon Jairo Bonilla; 3: Walter Sisquiarco; 4: Adrián Ríos; 5: Óscar Bustamante; 6: Germán Hoyos; 7: Rafael Mesa; 8: Mery Osorno; 9: Gloria Zabala) de la cuenca del río Porce. 
regula el microclima, proporciona refugio y hábitat para enemigos naturales y garantiza servicios ecosistémicos como la polinización, el control de la erosión y la captura de carbono (Poveda et al., 2001; Frank y Pennrose Buckley, 2012; Meylan et al., 2013; Souza et al., 2012). Cuatro de los nueve agricultores poseen sistemas de café bajo semisombrío asociado con guamo (Inga spp.), fijador de nitrógeno y nogal cafetero (Cordia alliodora).

En los últimos años se ha hecho énfasis en el deterioro de la biodiversidad en la conversión del café de sombra al café de sol. Varios estudios han demostrado que los elementos de biodiversidad son más altos en la sombra que en el café sin sombra (Cenicafé, 2008; Borkhataria et al., 2012). La conservación de la biodiversidad brinda servicios ecológicos a los agricultores en forma de control de plagas. Estudios han demostrado que las hormigas, aves y lagartos disminuyen la abundancia de insectos-plagas en el café (Perfecto et al., 2004; Borkhataria et al., 2012). La combinación de los objetivos de la agricultura y la conservación de la biodiversidad es prometedora para la conservación de la diversidad biológica en los trópicos (Perfecto et al., 1996). El aumentar la diversidad de los agroecosistemas por medio de la implementación de árboles para sombrío en el café traería ciertos beneficios económicos.
Solamente dos de los nueve agricultores carecían de bosques dentro de sus fincas, por lo que obtuvieron valores bajos en el indicador de vegetación natural circundante. Los remanentes de bosque son hábitat de plantas nativas; muchos animales usan los cafetales con sombra de forma ocasional, pero necesitan bosques para sobrevivir. Los árboles proporcionan alimento y refugio para la fauna y proveen recursos para una gran diversidad de aves. Al preservar la vegetación en las riberas de las cañadas y cauces de ríos y quebradas se conservan estas fuentes. Adicionalmente los árboles abastecen a las familias de recursos maderables, bajo un uso planificado y responsable, y son refugio de varias especies de insectos que contribuyen a la polinización en los cultivos. Algunos árboles contribuyen al control de insectos-plagas y ejercen protección contra la erosión y enriquecen estéticamente el paisaje cafetero (Cenicafé, 2012).

\section{Dimensión social}

En la Figura 3 se encuentran los resultados obtenidos en los atributos referidos a tenencia de la tierra y redes sociales. Los valores para la tenencia de la tierra fueron altos porque todos son propietarios, con excepción de los agricultores 2 y 9 que tienen la posesión pero no las escrituras. La tenencia de la tierra es uno de los elementos

Evaluación social en nueve fincas del nordeste antioqueño

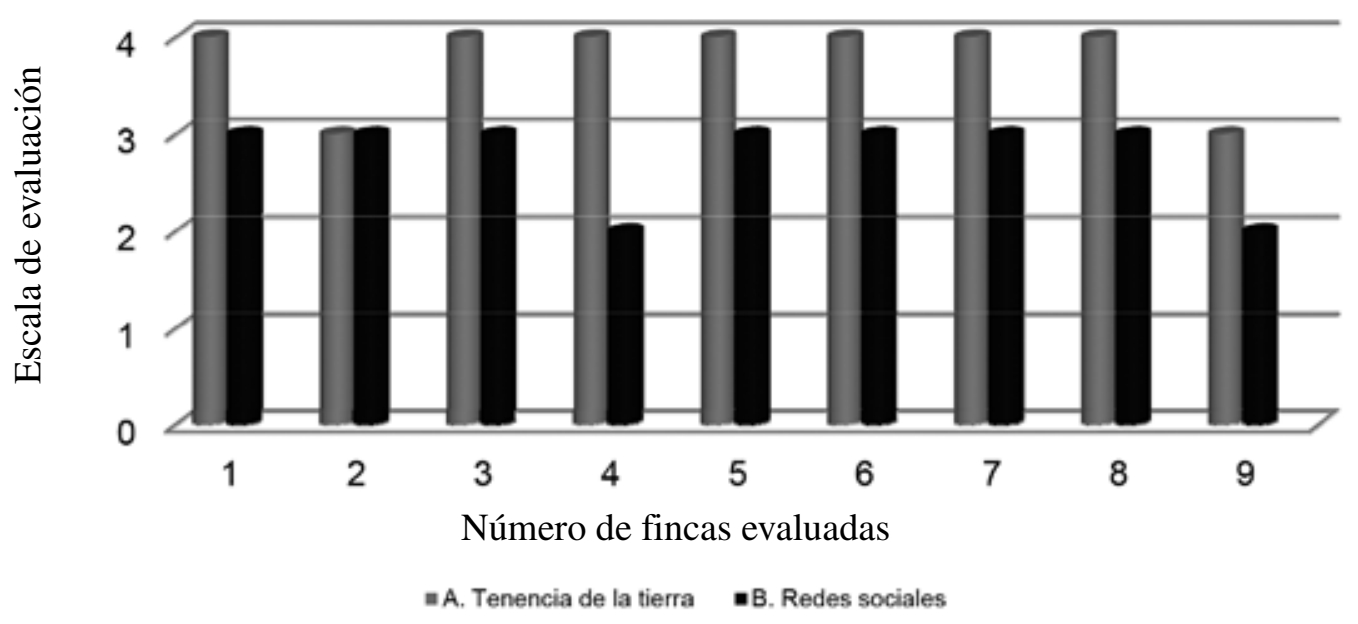

Figura 3. Caracterización de la dimensión social de los nueve agroecosistemas de café (1: Fabián Bonilla, 2: Jhon Jairo Bonilla; 3: Walter Sisquiarco; 4: Adrián Ríos; 5: Óscar Bustamante; 6: Germán Hoyos; 7: Rafael Mesa; 8: Mery Osorno; 9: Gloria Zabala) de la cuenca del río Porce. 
básicos de la estructura agraria y es esencial para la supervivencia del campesinado. Si bien el acceso a la tierra es necesario para reducir la pobreza rural, acceder al capital es igualmente importante para asegurar la competitividad de la agricultura familiar campesina. Un punto a favor de los agricultores estudiados es la tenencia de tierra propia. Solamente en dos casos no se habían legalizado las escrituras y esto les impedía a los agricultores acceder a créditos del Banco Agrario.

Los agricultores 4 (Adrián Ríos) y 9 (Gloria Zabala) obtuvieron los valores más bajos para redes sociales, esto se debe a que ninguno tiene parentela en la vereda y el apoyo institucional que reciben es escaso. Las redes sociales que se crean con la parentela, los lazos de vecindad, las juntas de acción comunal y las juntas administradoras de los acueductos rurales intensifican los mecanismos de reciprocidad y de cooperación y sirven de respaldo en momentos de crisis.

\section{Puntos críticos de los agroecosistemas de café}

En la Figura 4 se puede observar que los puntos críticos generales de los nueve agroecosistemas de café, es decir, aquellos evaluados con valores de 1 a 2 , son el rendimiento en la producción de café y el riesgo económico.

En la Figura 5 se observan los resultados de la caracterización del agroecosistema de café del municipio de Santo Domingo. El punto más crítico, con un valor 2 , es el rendimiento del café.

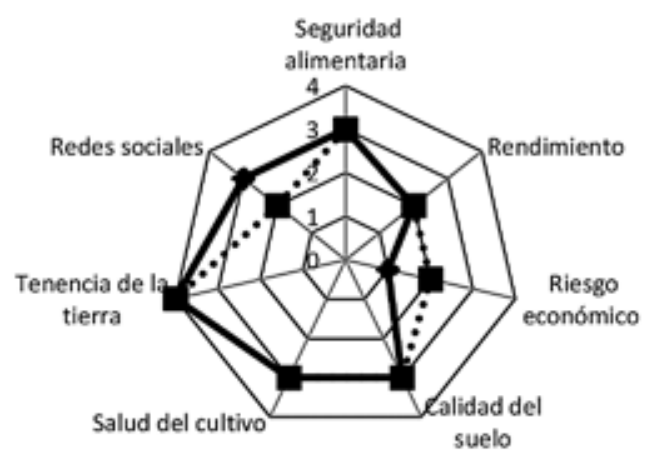

$\longrightarrow$ Walter S. ....... Adrian R.

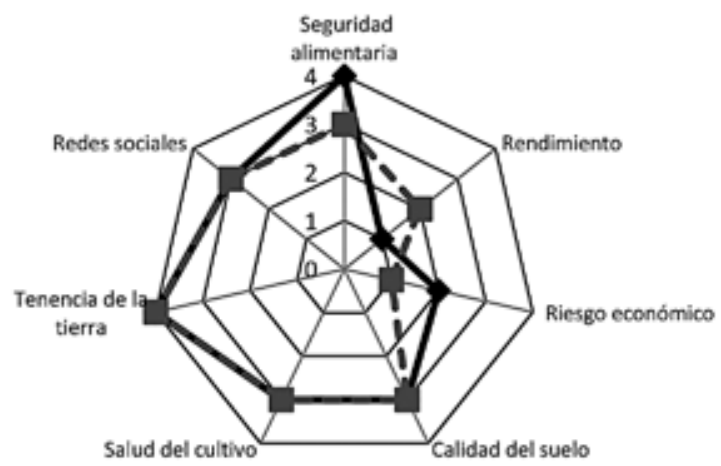

Germán H. - a - Rafael M.

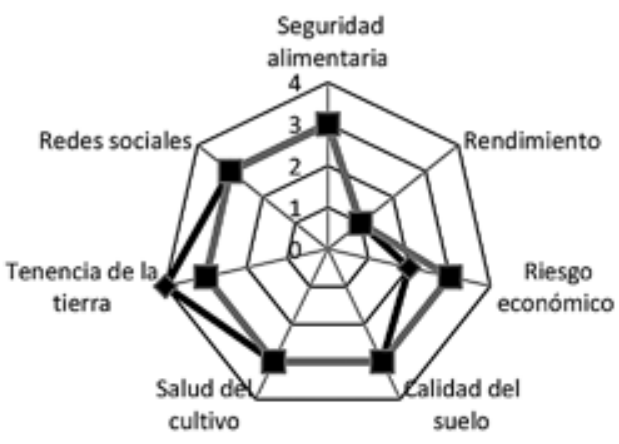

$\longrightarrow$ Fabián B. $\rightarrow$ - Whon Jairo B.

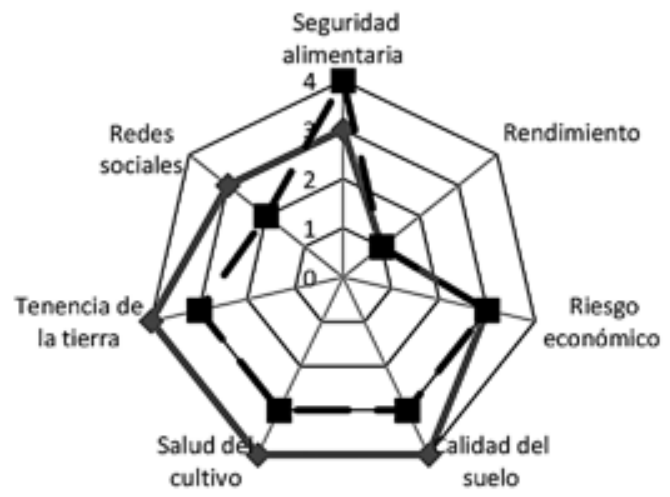

$\longrightarrow$ Mery O. $\rightarrow$ Gloria Z.

Figura 4. Puntos críticos de 8 agroecosistemas evaluados por medio de indicadores para la caracterización, municipio de Gómez Plata, Amalfi, Santa Rosa de Osos y Yolombó. 


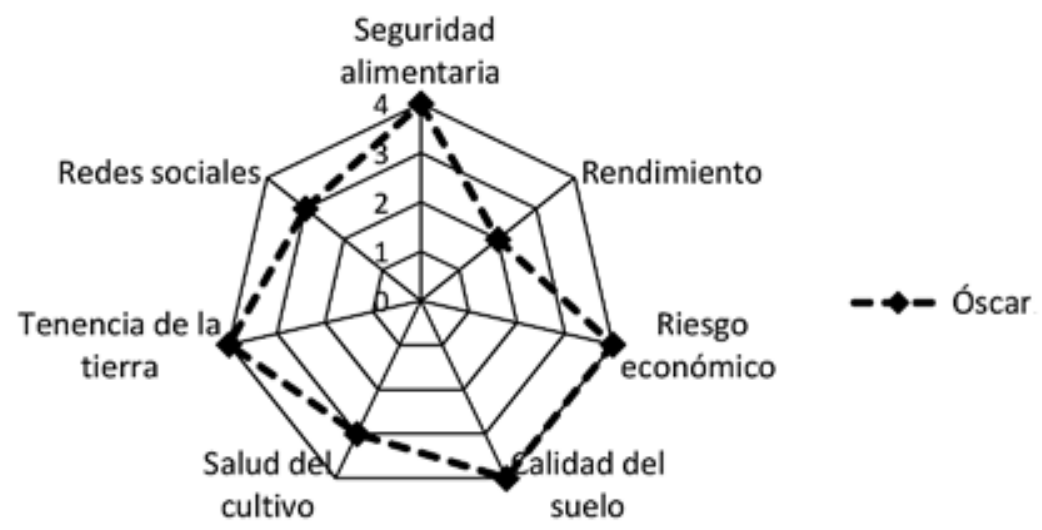

Figura 5. Puntos críticos del agroecosistema del municipio de Santo Domingo, evaluado por medio de indicadores para la caracterización.

Estos agricultores podrían aprovechar los desechos domésticos, los residuos de cosecha y la cascarilla de café para hacer compost y de esta forma abonar los cultivos y depender menos de los insumos externos. También les ayudaría realizar curvas a nivel para conservar el suelo en los cultivos de ladera. Para aumentar la productividad se requiere renovar los cafetales con variedades nuevas y hacer un rediseño del arreglo vegetal que tiene como cultivo principal el café, partiendo del sistema bajo sombrío con árboles maderables, fijadores de nitrógeno, árboles frutales y plátano, de esta forma se aumentan las interacciones biológicas, la sinergia del sistema y los servicios ecosistémicos. Al disponer de más productos para la venta y abrir otras vías de comercialización, como los mercados de cafés especiales, se disminuye el riesgo económico.

\section{Conclusiones}

El principal aporte de esta investigación se centra en el desarrollo de una metodología para la caracterización integral de un grupo de unidades agrícolas familiares productoras de café, que tiene en cuenta las dimensiones técnico-productiva, la económica y la social y que se realiza con la participación activa de los agricultores. Dicha metodología podrá ser utilizada por los investigadores en agroecología que deban caracterizar y tipificar los sistemas de producción agropecuario de una zona de estudio. La metodología permitió identificar como puntos críticos el rendimiento en la producción y el riesgo económico, ello indica que estos factores constituyen las principales limitantes para transformar el sistema cafetero hacia una producción sostenible. Los resultados de la caracterización hacen necesario implementar un diseño con enfoque agroecológico para incrementar la productividad del café y la estabilidad del agroecosistema. Esto contribuiría a mejorar la calidad de vida de los agricultores que ahora se ve amenazada por la dependencia de un solo producto comercializable, con altos costos de producción y sujeto al vaivén de los precios en el mercado internacional. Un rediseño agroecológico permitiría que estas familias tengan mayor resiliencia en un contexto de crisis socioeconómica del sector cafetero.

\section{Agradecimientos}

Los autores agradecen el apoyo económico del proyecto "La hacienda Vegas de La Clara como faro agroecológico para el nordeste antioqueño" financiado por la Universidad de Antioquia. Así mismo expresan su reconocimiento al proyecto de sostenibilidad 2013-2014 otorgado al Grupo de Investigación Medio Ambiente y Sociedad por la vicerrectoría de investigaciones de la misma institución. 


\section{Literatura Citada}

Altieri, M.A.

1999. Agroecología, Bases Científicas para una Agricultura Sustentable. Montevideo: Nordan- Comunidad. pp 325.

Altieri, M.A.

2002. Agroecología: principios y estrategias para diseñar sistemas agrarios sustentables. En: Sarandón, S. (Ed.) Agroecología: El Camino hacia una Agricultura Sustentable. Ediciones Científicas Americanas, Buenos Aires, pp. 49-56.

Altieri, M.A.; Nicholls, C.I.

2002. Un método agroecológico rápido para la evaluación de sostenibilidad en cafetales. Manejo Integrado de Plagas y Agroecología, 64:19-24.

Astier, M.; Masera, R.O.; Galván-Miyoshi, Y.

2008. Evaluación de sustentabilidad. Un enfoque dinámico y multidimensional. Seae, Ciga, Ecosur, Cieco, Unam, Gira, Mundiprensa, Fundación Instituto de agricultura ecológica y sustentable, España, Valencia. pp 200.

Álvarez, M.C.; López, M.; Cortés, L.P.; Torres, J.E.

2007. Caracterización socioecómica y seguridad alimentaria de los hogares productores de alimentos para el autoconsumo, Antioquia-Colombia. Agroalimentaria, 25: 109-122.

Borkhataria, R.; Collazo, J.A.; Groom, M.J.; Jordan-García A. 2012. Shade-grown coffee in Puerto Rico: Opportunities to preserve biodiversity while reinvigorating a struggling agricultural commodity. Agriculture, Ecosystems and Environment, 149: 164-170.

Carneiro, T.J.; Conceção, P.C.; Bayer, C.; Foletto, F.L. 2007. Qualidade do solo avaliada "Soil quality kit test" em dois experimentos de longa duração no Rio Grande do sul. R. Bras. Ci. Solo, 31: 109121.

Castro-Tanzi, S.; Dietsch, T.; Urena, N.; Vindas, L.; Chandler, M. 2012. Analysis of management and site factors to improve the sustainability of smallholder coffee production in Tarrazú, Costa Rica. Agriculture, Ecosystems and Environment, 155: 172-181.

Cenicafé

2008. Estudios regionales de biodiversidad en las zonas cafeteras de Colombia. Avance Técnico, 378: 2-8.

Cenicafé

2009. Variabilidad climática en la zona cafetera colombiana asociada al evento del El Niño y su efecto en la caficultura. Avances Técnicos, 390: 2-8.

Cenicafé

2010. Café orgánico al sol y bajo sombrío. Una doble posibilidad para la zona cafetera de Colombia. Avances Técnicos, 399: 2-8.

Cenicafé

2012. Herramientas de manejo del paisaje para la conservación de la biodiversidad. Avances Técnicos, 416: 2-12.

Conceção, P.C.; Carneiro, T.J.; Mielniczuk, J.; Spagnollo, E.

2005. Qualidade do solo em sistemas de manejo avaliada pela dinâmica da matéria orgânica e atributos relacionados. $R$. Bras. Ci. Solo, 29: 777-788.

CTA (Centro de Tecnología de Antioquia); COSUDE (Agencia Suiza para el desarrollo y la Cooperación)

2013. Resumen de resultados. Evaluación de la huella hídrica en la cuenca del río Porce, 2013. En: cta.org.co/ wp-content/uploads/2013/05/librohh.pdf , consultado: 8/ Nov./ 2013.
De Schutter, O.

2010. Informe del relator especial sobre el derecho a la alimentación. Naciones Unidas, Asamblea General, 20 de diciembre, pp. 24.

Fernández, C.E.; Muschler, R.

1999. Aspectos de sostenibilidad de los sistemas de cultivo de café en América Central, En: Desafíos de la caficultura en Centro América. B,Bertrand (ed). IICA-PROMECAFECIRAD, San José, Costa Rica: 69-96.

Frank, J.; Pennrose Buckley, C.

2012. Small-scale farmers and climate change. How can farmer organisations and Fairtrade build the adaptive capacity of samllholders? IIED, Londres, pp. 31.

Gliessman, S. 1998. Agroecology: ecology processes in sustainable agriculture, ANNN Arbor Press, Michigan, pp. 369.

Hart, R.D.

1985. Conceptos básicos sobre agroecosistemas. CATIE, Turrialba, Costa Rica, pp. 160.

Holdridge, L.R.

1978. Ecología basada en zonas de vida. IICA, San José, Costa Rica, pp. 216.

Instituto de Hidrología, Meteorología y Estudios Ambientales (IDEAM)

2012. Boletín informativo sobre el monitoreo de los Fenómenos de variabilidad climática "El Niño" y "La Niña". Boletín $\mathrm{N}^{\mathrm{o}}$ 50: 5 pp.

Instituto de Hidrología, Meteorología y Estudios Ambientales (IDEAM)

2013. Boletín informativo sobre el monitoreo de los Fenómenos de variabilidad climática "El Niño" y "La Niña”. Boletín $\mathrm{N}^{\mathrm{o}}$ 53: 4 pp.

Jaramillo, D.

1989. Estudio general de suelos, erosión y uso potencial agropecuario para los proyectos hidroeléctricos Porce II y Porce III. Empresas Públicas de Medellín, Colombia, pp. 99.

Khalajabadi, S.S.

2008. Fertilidad del suelo y nutrición del café en Colombia. Chinchiná, Cenicafé, pp. 44 (Boletín Técnico No 32).

Masera O., Astier, M.; López-Ridaura, S.

1999. Sustentabilidad y manejo de recursos naturales: el marco de evaluación MESMIS. Mundiprensa, GIRA, UNAM, México D.F.: 160 pp.

Meylan, L.; Merot, A.; Gary, C.; Rapidel, B.

2013. Combining a typology and a conceptual model of cropping system to explore the diversity of relationships between ecosystem services: The case of erosion control in coffee-based agroforestry systems in Costa Rica. Agricultural Systems, 118: 52-64.

Ortiz, S. (eds.)

Integrating agriculture, conservation and ecoturism: examples from the field. Springer. Netherlands, pp. 141-208.

Pereira, R.; Quacchia, S.A.

2009. Desafios na construção de indicadores de sustentabilidade. Rev. Ambiente \& Sociedade. Campinas, XII (2): 307-323.

Perfecto, I.; Vandermeer, J.H.; Bautista, G.L.; Nunez, G.I.; Greenberg, R.; Bichier, P.; Langridge, S.

2004. Greater predation in shaded coffee farms: the role of resident neotropical birds. Ecology, 85 (10): 2677-2681. 
Perfecto, I.; Rice, R.

1996. Shade coffee: a dissapearing refuge for biodiversity. Bioscience 46 (8): 598-608.

Poveda, G.; Jaramillo, Á.; Gil, M.M.; Quiceno, N.; Mantilla R. 2001. Seasonality in ENSO-related precipitation, river discharges, soil moisture, and vegetation index in Colombia. Water Resources Research, 37 (8): 2169-2178.

Rosset, P.; Collins, J.; Moore Lapp, F.

2000. Lecciones de la revolución verde. Boletín de la Reb Alerta sobre transgénicos, 2 (15): 1-8.

Sarandón, S.J.

2002. El desarrollo y uso de indicadores para evaluar la sustentabilidad de los agroecosistemas. En: Agroecología: El camino hacia una agricultura sustentable. Ediciones Científicas Americanas. Argentina: 350-394.

Sarandón, S.J.; Zuluaga, M.S.; Cieza, R.; Gómez, C.; Janjetic, L.; Negrete, E.

2006. Evaluación de la sustentabilidad de sistemas agrícolas de fincas en Misiones, Argentina, mediante el uso de indicadores. Revista Agroecología 1: 19-28.
Sarandón, S.J.; Flores, C.C.

2009. Evaluación de la sustentabilidad en agroecosistemas: una propuesta metodológica. Agroecología , 4: 19-28.

Souza, H. N. de, de Goede, R.G.M.; Brussaard, L.; Cardoso, I.M.; Duarte, E.M.G.; Fernandes, R.B.A.; Gomes, L.C.; Pulleman, L.L.

2012. Protective shade, tree diversity and soil properties in coffee agroforestry systems in the Atlantic Rainforest biome. Agriculture, Ecosystems and Environment 146: 179-196.

Uribe de Hincapie, M.T.; Álvarez, J.M.

1985. Minería, comercio y sociedad en Antioquia 1760-1800. Lecturas de Economía, 18: 53-113.

Youkhana, A.; Idol, T.

2009. Tree pruning mulch increases soil $\mathrm{C}$ and $\mathrm{N}$ in a shaded coffee agroecosystem in Hawaii. Soil Biology \& Biochemistry, 41: $2527-2534$. 
